\title{
Crescimento em diâmetro do Pinus elliottii e Pinus taeda em áreas arenizadas e degradadas no Oeste do Rio Grande do Sul
}

\author{
Diameter growth of Pinus elliottii and Pinus taeda in arenizated and \\ degraded areas in the Western of Rio Grande do Sul State, Brazil
}

\author{
Paulo Renato Schneider ${ }^{I^{*}}$ Luiz Ernesto Grilo Elesbão ${ }^{\mathrm{I}}$ \\ Paulo Sérgio Pigato Schneider ${ }^{\mathrm{II}}$ Régis Villanova Longhi ${ }^{\mathrm{II}}$
}

\section{RESUMO}

Este trabalho foi realizado com o objetivo de estudar o desempenho do Pinus elliottii Engelm. e Pinus taeda L. em áreas arenizadas e degradadas por ação antrópica, na região da fronteira oeste do estado do Rio Grande do Sul. Em povoamentos, foram selecionadas árvores médias, que foram abatidas e seccionadas pelo método de Smalian, a fim de obter discos de madeira para a análise dendrocronológica, para a obtenção do diâmetro por idade. O crescimento em diâmetro, quando comparado entre espécies, apresentou tendências diferentes de níveis de desenvolvimento no tempo. O uso de variáveis dummy no modelo de crescimento permitiu definir uma equação única para estimar o diâmetro em função da idade para ambas as espécies, considerando os níveis de degradação do solo médio e baixo, como variáveis necessárias para estimar o crescimento destas espécies nestes tipos de solos arenizados e degradados.

Palavras-chave: performance, funções de crescimento, arenização, degradação do solo.

\section{ABSTRACT}

This research aims to study the performance of Pinus elliottii Engelm. and Pinus taeda L. in the arenizated and degraded areas by antropic action in the western of Rio Grande do Sul state. In stands were selected medium trees, which were harvested and sectioned by Smalian method to obtain wood discs for dendro chronological analysis to determine the diameter by age. The diameter growth compared between species showed different levels of development over time. The use of dummy variables in the growth model enables a single equation to estimate the diameter as a function of age for both species, considering the medium and low levels of soil degradation, as variables necessary to estimate the growth of these species in these types of arenizated degraded soils.

Key words: performance, growth functions, arenization, soil degradation.

\section{INTRODUÇÃO}

A formação dos areais, no estado do Rio Grande do Sul, interpretada a partir de estudos geomorfológicos, está associada à dinâmica dos fatores hídricos e eólicos, que, relacionados à topografia favorável, permitem, numa primeira fase, a formação de areais, com ravinas e voçorocas. Por outro lado, em decorrência do processo de transporte de sedimentos pela água durante episódios de chuvas torrenciais, formam-se depósitos arenosos em forma de leques. Com o tempo, esses leques vão agrupando-se e, em conjunto, dão origem a um areal, onde o vento, atuando sobre esses areais, em todas as direções, permite a ampliação desse processo (SUERTEGARAY, 2011).

A United Nations Covention to Combat Desertification (UNCCD, 1994) definiu desertificação como sendo a degradação do solo em regiões áridas, semiáridas e de áreas secas a subúmidas, resultante de vários fatores, incluindo a variação climática e atividades humanas. Considerando-se estes aspectos, percebe-se que o Rio Grande do Sul não se apresenta como região afetada pela desertificação, pois a precipitação média anual é em torno de $1.400 \mathrm{~mm}$.

Na Conferência das Nações Unidas sobre Desertificação, realizada em 1977, em Nairóbi, Quênia, o termo desertificação foi conceituado como sendo a diminuição ou a destruição do potencial biológico do solo, que pode desembocar,

\footnotetext{
'Departamento de Ciências Florestais, Centro de Ciências Rurais (CCR), Universidade Florestal de Santa Maria (UFSM), 97105-900, Santa Maria, RS, Brasil. E-mail: schneider.paulorenato@gmail.com. *Autor para correspondência.

IIPrograma de Pós-graduação em Engenharia Florestal, CCR, UFSM, Santa Maria, RS, Brasil.
} 
em definitivo, na condição do tipo desértico. Além do plano de ação, foi elaborado um mapa mundial com a localização dos desertos e das áreas de risco à desertificação, classificados em muito alta, alta e moderada, em que, no Brasil, somente o nordeste é incluído (ONU, 2005).

Nessas áreas, constata-se um potencial para a atividade florestal, principalmente nos municípios de São Francisco de Assis, Alegrete e Manoel Viana e municípios limítrofes, visto que, nestas áreas impróprias para a agricultura, é possível implantar florestas, que agem como protetoras dos solos frágeis, e adotar práticas de exploração racional. O cultivo de espécies florestais de crescimento rápido é lucrativo e, muitas vezes, mais vantajoso do que os cultivos agrícolas, particularmente em solos mais pobres. Sob essa ótica, os gêneros Eucalyptus e Pinus já demonstraram viabilidade para as condições locais, constituindo-se em importantes referências para plantios comerciais (FREITAS et al., 2003).

O crescimento das árvores em geral aumenta com a precipitação (MURPHY \& LUGO, 1986) e diminui com a seca (NATH et al., 2006), mas, independentemente da chuva, a fertilidade do solo pode afetar a taxa de crescimento das árvores (LU et al., 2002; MALHI et al., 2004), obtendo-se um maior crescimento em solos mais ricos em nutrientes (RUSSO et al., 2005). Por outro lado, a disponibilidade de água da área depende da posição topográfica em que ela se encontra, pois influencia diretamente o desenvolvimento das plantas do local, assim como a formação do solo (HANNAH et al., 1982).

A produtividade de Pinus taeda está positivamente associada aos solos de maior fertilidade, com maiores teores de nitrogênio e fósforo (REISSMANN \& WISNEWSKI, 2005). Sendo que, nos sítios de maior produtividade, as árvores tendem a ser mais uniformes do que em sítios pobres, tendendo a diminuir, considerando a mesma idade (BINKLEY et al., 2010). As espécies de Pinus demonstram capacidade extraordinária de gerenciamento dos recursos nutricionais em sítios de baixa fertilidade, sem, no entanto, manifestar sintomas visuais claros de deficiência. Esse aspecto, embora positivo sob um determinado ponto de vista, gera uma expectativa que se mostra negativa, no sentido do manejo nutricional. A rapidez de crescimento e ausência de sintomas de deficiência, especialmente durante as primeiras rotações, o que reforça a expectativa de que os Pinus, em geral, dispensariam grandes cuidados com a adubação, ou que esta fosse totalmente dispensável (REISSMANN \& WISNEWSKI, 2005).
Assim, o objetivo geral deste trabalho foi estudar o crescimento em diâmetro por idade das espécies Pinus elliottii e Pinus taeda implantadas em áreas de solos arenizadas, em diferentes níveis de degradação, na região da fronteira oeste do estado do Rio Grande do Sul.

\section{MATERIAL E MÉTODOS}

Este estudo foi realizado em áreas arenizadas localizadas nos municípios de Alegrete e São Francisco de Assis. Estes locais encontram-se situados na região da fronteira oeste do estado do Rio Grande do Sul, numa latitude de $29^{\circ} 47^{\prime} 01,63^{\prime \prime}$ Sul e longitude de 5547’27,54”' Oeste.

O clima da região do estudo, de acordo com a classificação de Köppen, recebe a denominação de Cfa1g, subtropical mesotérmico úmido (NIMER, 1990), sendo caracterizado por meses de frio, com geadas de maio a agosto, e calor intenso nos meses de janeiro e fevereiro, sendo a temperatura média dos meses mais quentes superior a $20^{\circ} \mathrm{C}$. As precipitações são bem distribuídas durante todo o ano, variam entre 1.250 e $1.500 \mathrm{~mm}$ ano $^{-1}$.

Segundo STRECK et al. (2008), na região do presente estudo ocorrem Latossolos Vermelhos e Neossolos Quartzarênicos, que apresentam alta suscetibilidade à erosão, notabilizando o processo de arenização nestas áreas.

A amostragem dos dados dendrométricos foi realizada nos dois municípios, perfazendo sete fazendas contendo áreas reflorestadas com Pinus elliottii e Pinus taeda e com características distintas de níveis de degradação do solo. Esses povoamentos florestais foram implantados em diferentes épocas, em espaçamento inicial variando de $3 \times 2 \mathrm{~m}$ a $3 \times 3 \mathrm{~m}$, com o objetivo inicial de amenização do processo de degradação e recuperação de solos arenizados. Estas áreas foram estratificadas por espécie e níveis de degradação do solo: Alto - áreas arenizadas sem presença de vegetação; Médio - áreas arenizadas com presença de tuchos de vegetação; Baixo - áreas arenizadas com presença de vegetação esparsa, baseado na classificação mencionada pela United Nations Covention to Combat Desertification UNCCD (1994). Nesses povoamentos, foram lançadas unidades amostrais permanentes de $20 \times 20 \mathrm{~m}$, sendo mensuradas todas as árvores, em relação ao diâmetro, à altura e à altura dominante, e feita a caracterização da posição sociológica, segundo a classificação de Kraft (ASSMANN, 1970). No momento da coleta dos dados os povoamentos apresentavam idade entre 24 a 29 anos, sem intervenção de desbaste. 
Para o estudo do crescimento em diâmetro do Pinus elliottii e Pinus taeda, nas unidades amostrais, foram selecionadas árvores representativas em seu estrato médio, as quais passaram por análise de tronco completa. Essas árvores amostradas pertenciam à classe sociológica intermediária, que forma a parte principal do povoamento, e apresentavam suas copas medianamente desenvolvidas. $\mathrm{Na}$ área de estudo, foram abatidas um total de 32 árvores médias distribuídas por espécie e níveis de degradação do solo, sendo respeitado o princípio da aleatoriedade. O nível de degradação alto foi amostrado, mas desconsiderado da análise estatística, devido à baixa densidade de ocorrência de reflorestamentos nestas áreas.

Na coleta de dados, foram utilizadas as normas para coleta de informações dendrométricas descritas por SCHNEIDER et al. (1988). As árvores amostradas foram seccionadas em toras no comprimento fixo. De cada árvore, foram extraídos discos na altura de 0,$1 ; 1,3 ; 3,3 ; 5,3 \mathrm{~m}$, e os demais, de 2 em 2 metros, até o ápice da árvore. Estes discos tinham cerca de $5 \mathrm{~cm}$ de espessura e foram secos em estufa e, posteriormente, preparados pelo lixamento, até a visualização integral de todos os anéis de crescimento. Em cada disco, foram tomadas medidas em quatro raios, sendo o primeiro num ângulo de $45^{\circ}$ a partir do maior raio da fatia, e os demais, a $90^{\circ}$ um do outro. Os anéis de crescimento foram identificados com auxílio de lupa, e suas espessuras, medidas com auxílio do Lintab II, acoplado a um computador.

A identificação dos anéis de crescimento foi realizada de acordo com a orientação de SCHWEINGRUBER (1996), que descreveu os problemas na identificação de anéis de crescimento anual em Pinus, afirmando que podem existir anéis muito tênues, próximos à medula, de difícil identificação visual. Também podem existir falsos anéis, causados por estresse, relacionando os fatores que influem na sua formação e algumas técnicas utilizadas para identificar anéis verdadeiros.

Para o estudo do crescimento em altura dominante em função da idade foram ajustados os seguintes modelos (RICHARDS, 1959; LOETSCH et al., 1973; SCHNEIDER, 1984; PRODAN et al., 1997):

Schumacher (1): 1n $y=\mathrm{b}_{0}+\mathrm{b}_{1} .(1 / t)$;

Backman (2): 1n $y=\mathrm{b}_{0}+\mathrm{b}_{1} \cdot 1 \mathrm{n} t+\mathrm{b}_{2} \cdot 1 \mathrm{n}^{2} t$;

Backman modificado (3): $1 \mathrm{n} y=\mathrm{b}_{1} \cdot 1 \mathrm{n} t+\mathrm{b}_{2} \cdot 1 \mathrm{n}^{2} t$;

Prodan (4): 1n $y=t^{2} /\left(\mathrm{b}_{0}+\mathrm{b}_{1} \cdot t+\mathrm{b}_{2} \cdot t^{2}\right)$;

Richards (5): $\mathrm{y}=\mathrm{b}_{0} .\left(1-\mathrm{e}^{\left(-\mathrm{b}_{1} . t\right)}\right)$; em que y é a variável dependente; $t$ a idade em anos; $b_{0}, b_{1}, b_{2}$ os parâmetros; ln é o logaritmo neperiano.

O método de análise de covariância foi utilizado para avaliar as diferenças de inclinação e nível entre as curvas de crescimento em diâmetro por idade entre as árvores médias de Pinus elliottii e Pinus taeda, considerando os níveis de degradação do solo. Na análise de covariância, utiliza-se, conjuntamente, os conceitos de análise de variância e regressão e relacionam-se as variáveis independentes, em que não se exerce um controle exato sobre elas (STEEL \& TORRIE, 1960). A análise de covariância foi determinada tomando por base de crescimento em diâmetro o modelo de Backman modificado. Nesse modelo, foram introduzidas as variáveis dummy para as espécies, indicadas pelo Pinus elliottii igual a 0 (presença) e pelo Pinus taeda igual a 1 (ausência) e para os níveis de degradação do solo médio igual a 0 (presença) e baixo igual a 1 (ausência).

O ajuste das regressões e análise de covariância para verificar as hipóteses de igualdade de inclinação e nível das curvas de crescimento em diâmetro foi efetuado através do teste $\mathrm{F}$, para $1 \%$ de erro, processadas no programa de computador Statistical Analysis System (SAS, 2004).

\section{RESULTADOS E DISCUSSÃO}

As análises dos dados de crescimento em diâmetro no tempo permitiram o ajuste dos modelos e a determinação da precisão estatística de cada equação testada. Foram determinados os coeficientes e os parâmetros estatísticos de cada equação, para posterior comparação da precisão e ajuste, sendo eles apresentados na tabela 1.

Analisando esses valores, percebe-se que, em geral, as equações apresentaram valores de coeficiente de determinação ajustado entre 0,63 e 0,97, e coeficientes de variação entre 17,08 e 26,94\%, variando com o modelo testado. Os valores de coeficiente de determinação discrepantes indicam que algumas equações têm maior capacidade de explicar a variação total do que outras, porém seu uso isolado pode provocar erros, já que a inclusão de uma única variável pode aumentar seu valor, favorecendo a sua escolha.

Ao analisar as equações através dos parâmetros estatísticos, destaca-se: a equação de número 4, definida pelo modelo de crescimento de Prodan, que apresentou o $3^{\circ}$ maior valor de coeficiente de determinação ajustado de 0,85 e coeficiente de variação de 26,94\%; o modelo de Chapman-Richards, representado pela equação 5, com coeficiente de determinação ajustado de 0,94 e coeficiente de variação de 25,94\%; e o modelo de Backman modificado, correspondente à equação 3, que apresentou o maior coeficiente de determinação ajustado de 0,97 e coeficiente de variação de 17,34\%. 
Tabela 1 - Parâmetros estatísticos das equações testadas para ajustar o crescimento de diâmetro em função da idade de dados agrupados de Pinus elliottii e Pinus taeda.

\begin{tabular}{|c|c|c|c|c|c|c|c|c|}
\hline \multirow[b]{2}{*}{ Equação } & \multicolumn{3}{|c|}{------------------ Coeficientes ------------------- } & \multirow[b]{2}{*}{$\mathrm{R}_{\mathrm{Aj}}^{2}$} & \multirow[b]{2}{*}{$\mathrm{S}_{\mathrm{yx}}$} & \multirow[b]{2}{*}{$\mathrm{CV} \%$} & \multirow[b]{2}{*}{$\mathrm{F}$} & \multirow{2}{*}{$\begin{array}{c}\text { Valor } \\
\text { Escores }\end{array}$} \\
\hline & $\mathrm{b}_{0}$ & $\mathrm{~b}_{1}$ & $\mathrm{~b}_{2}$ & & & & & \\
\hline 1 & 2,7156 & $-7,9629$ & - & $0,63(5)$ & $0,3764(3)$ & $18,45(3)$ & $441,13 * *(4)$ & (15) \\
\hline 2 & $-0,9686$ & 1,4485 & $-0,1136$ & $0,69(4)$ & $0,3484(1)$ & $17,08(1)$ & $278,66 * *(5)$ & (11) \\
\hline 3 & - & 0,6525 & 0,0419 & $0,97(1)$ & $0,3537(2)$ & $17,34(2)$ & $4422,51^{* *}(1)$ & (06) \\
\hline 4 & 23,0175 & 1,5967 & 0,2941 & $0,85(3)$ & $39,8998(5)$ & $26,94(5)$ & $718,14^{* *}(3)$ & (16) \\
\hline 5 & 29,1305 & 0,0232 & - & $0,94(2)$ & $2,3298(4)$ & $25,94(4)$ & $2148,71^{* *}(2)$ & (12) \\
\hline
\end{tabular}

Sendo: $\mathrm{R}^{2}{ }_{\mathrm{Aj}}=$ coeficiente de determinação ajustado; $\mathrm{S}_{\mathrm{yx}}=$ erro padrão da estimativa; ** = significativo a $95 \%$ de probabilidade; $\mathrm{CV} \%=$ coeficiente de variação em \%; $F=$ valor de $F$ da análise de variância; e $b_{0}, b_{1}, b_{2}=$ coeficientes; ( ) = escore dos parâmetros estatísticos.

A escolha da equação foi realizada pela determinação do valor dos escores estatísticos (Tabela 1). Com este procedimento estatístico, buscou-se sistematizar os resultados para auxiliar na seleção do modelo para descrever o crescimento do diâmetro em função da idade, sendo a equação selecionada o modelo de Backman modificado, pois apresentou o menor valor dos escores estatísticos, confirmando-a como a mais adequada para determinar o crescimento em diâmetro no tempo com dados agrupados de Pinus elliottii e Pinus taeda.

Os resultados da análise de covariância do crescimento em diâmetro em função da idade tiveram como co-variáveis as duas espécies e os dois níveis de degradação do solo. Observou-se que as espécies de Pinus elliottii e Pinus taeda apresentaram comportamento de crescimento diamétrico diferente, com um valor de $\mathrm{F}$ igual a 40,17, significativo (Prob. $<0,0001$ ), indicando a existência de níveis diferenciados de crescimento em diâmetro por idade. Da mesma forma, o crescimento em diâmetro em função da idade em relação aos níveis de degradação do solo apresentou um valor de F igual a 27,63, significativo (Prob.<0,0001), o que indica haver diferença na velocidade de crescimento em diâmetro por idade das árvores, sendo influenciado direta e fortemente pelos níveis de degradação do solo.

Esses resultados da análise de covariância do diâmetro em função da idade, considerando as relações entre espécies e os níveis de degradação do solo, indicam claramente que esta variável deve ser estimada por espécie, considerando a variação da capacidade produtiva do solo, definido pelos níveis de degradação, não permitindo o agrupamento de dados dendrométricos para o ajuste de funções de crescimento do diâmetro em função da idade.

Para estimar o diâmetro em função da idade para as espécies de Pinus elliottii e Pinus taeda de forma individualizada, plantados em solos arenizados e degradados da região da fronteira oeste do Rio Grande do Sul, foi introduzida no modelo uma variável dummy, cuja análise de variância por soma de quadrados do Tipo I é apresentada na tabela 2.

Nessa tabela, observa-se que todos os parâmetros do modelo apresentaram um valor de $\mathrm{F}$ significativo, tanto para espécie como para o nível de degradação do solo, justificando a sua permanência no modelo original, para a estimativa do diâmetro no tempo, de forma simultânea. A interação da idade com a espécie (ln t. D1), definido pela variável dummy 1, não foi significativa, com um valor de $\mathrm{F}$ igual a 3,24 (Prob.=0,0744), tendo sido excluída do modelo geral. Também, para o nível de degradação do solo a interação da idade com o nível de degradação do solo (ln t. D2), definido pela variável dummy 2 , não foi significativa, com um valor de $\mathrm{F}$ igual a 1,26 (Prob.=0,2632), sendo igualmente excluída do modelo geral. Desta análise, desprende-se que estas duas espécies apresentam diferença de níveis, porém com a mesma inclinação do crescimento em diâmetro no tempo, condicionadas à influência direta dos níveis de degradação do solo.

Os coeficientes do modelo de Backman modificado reduzido, resultantes da análise estatística, foram significativos, com probabilidade elevada para todas as variáveis consideradas. Assim, estes resultados permitiram definir um modelo único para estimar o diâmetro por idade, considerando simultaneamente a espécie e o nível de degradação do solo. Este modelo geral passou a ser expresso por: $\ln d=0,7729 . \ln \mathrm{t}-0,2059$. D1 + 0,1784 . D2

Esse modelo de crescimento em diâmetro (d), expresso na equação de Backman modificada, é uma função da idade (t), com inclusão da variável dummy (D1), representando as espécies, que assume 0 na presença de Pinus elliottii e 1 na presença de 
Tabela 2 - Análise de covariância com inclusão de variável dummy do crescimento em diâmetro das espécies de Pinus elliottii e Pinus taeda e nível de degradação do solo.

\begin{tabular}{|c|c|c|c|c|c|}
\hline FV & GL & SQ & $\mathrm{QM}$ & $\mathrm{F}$ & Prob. $>\mathrm{F}$ \\
\hline Modelo & 6 & 36,2971 & 6,0495 & 55,81 & $<, 0001$ \\
\hline $\ln t$ & 1 & 33,0529 & 33,0529 & 304,92 & $<, 0001$ \\
\hline D1 & 1 & 1,4626 & 1,4626 & 13,49 & 0,0004 \\
\hline $\ln t * D 1$ & 1 & 0,3514 & 0,3514 & 3,24 & 0,0744 \\
\hline D2 & 1 & 1,1612 & 1,1612 & 10,71 & 0,0014 \\
\hline $\ln t * D 2$ & 1 & 0,137 & 0,137 & 1,26 & 0,2632 \\
\hline Resíduo & 117 & 12,6824 & 0,1083 & - & - \\
\hline Total & 123 & 48,9796 & - & - & - \\
\hline
\end{tabular}

Sendo: $\mathrm{FV}$ = fonte de variação; $\mathrm{GL}=$ graus de liberdade; $\mathrm{SQ}=$ soma de quadrados; $\mathrm{QM}=$ quadrado médio; $\mathrm{F}=$ valor de $\mathrm{F}$; Prob. $>\mathrm{F}=$ probabilidade de significância para o valor de F; ln = logaritmo natural; t = idade; D1 = Dummy da espécie; D2 = Dummy da degradação do solo.

Pinus taeda; e da variável dummy (D2), representando os níveis de degradação do solo, que assume 0 na presença de nível médio de degradação e 1 na presença de nível baixo de degradação. Este modelo apresentou um excelente ajuste, com coeficiente de determinação igual a 0,97 , baixo erro padrão da estimativa, igual a 0,3319, e alta significância, com valor de F igual a 3.359,34.

Com a equação de Backman modificada, ajustada com variável dummy, estimaram-se os diâmetros por idade para Pinus elliottii (dummy $=0$ ) e Pinus taeda (dummy $=1$ ) e o nível de degradação médio $($ dummy $=0)$ e baixo $($ dummy $=1)$. Esses dados de crescimento em diâmetro por idade, estimados para estas duas espécies, estão representados na figura 1. Observa-se que o desenvolvimento do diâmetro no tempo, do Pinus elliottii, apresenta um melhor desempenho do que o Pinus taeda, considerando os mesmos tipos de solos arenizados e degradados.

Pode-se se inferir também que os diâmetros por idade das árvores médias, obtidos nestes solos degradados e arenizados da fronteira oeste do RS, em termos quantitativos, são muito inferiores aos convencionalmente encontrados em outras regiões

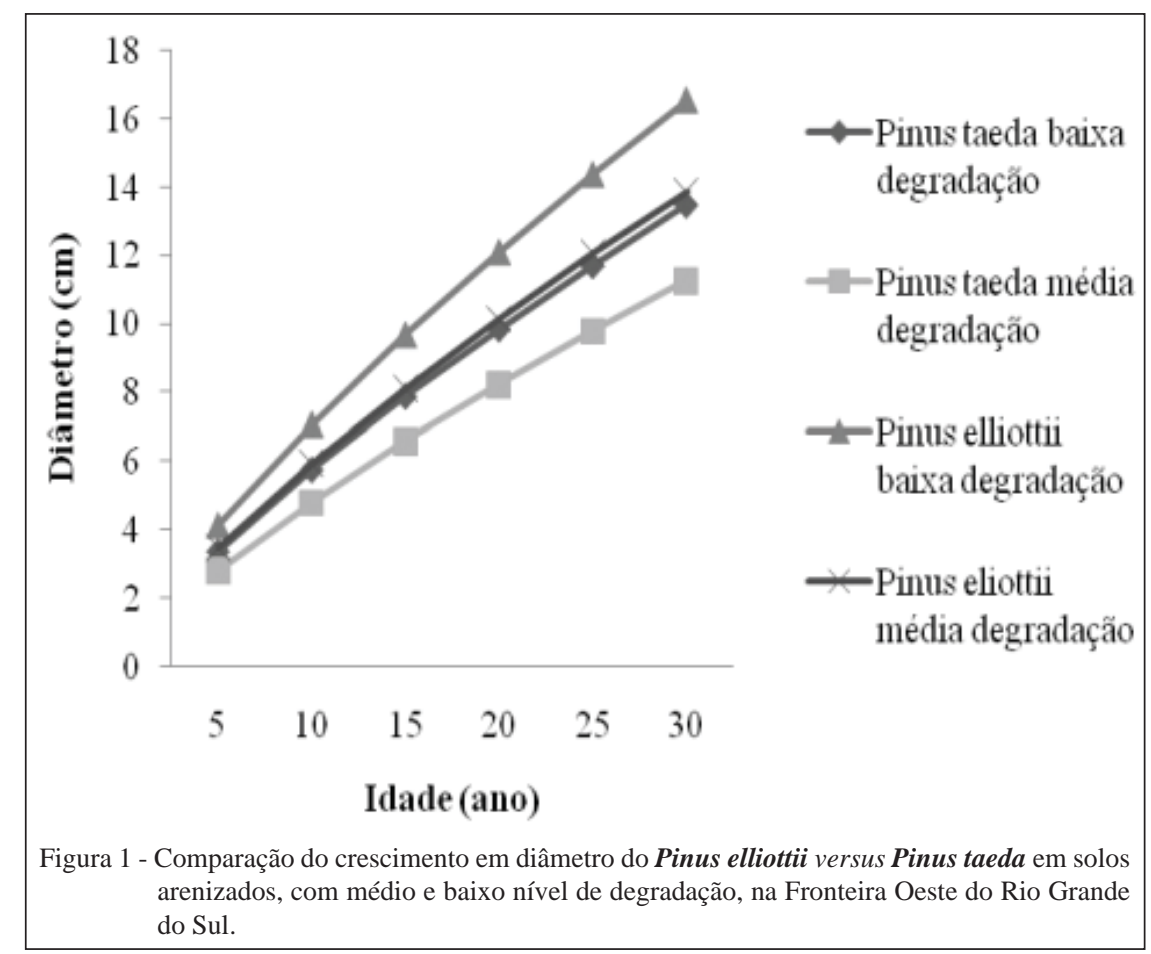

Ciência Rural, v.44, n.9, set, 2014. 
do Rio Grande do Sul, principalmente devido à disponibilidade de água desses solos, sendo o fator que mais determina o crescimento das árvores. Para GONÇALVES et al. (1990), as propriedades físicas do solo têm se relacionado, com maior frequência, à capacidade produtiva dos sítios florestais do que as químicas, principalmente nos aspectos da capacidade de retenção e armazenamento de umidade do solo.

Da mesma forma, GARICOITS (1990), analisando o crescimento de Pinus em solos derivados de três materiais de origem diferentes, verificou que as características dos materiais de origem têm afetado as características físicas e químicas e possivelmente a disponibilidade de água dos solos, fazendo com que, nos solos sobre arenito, aconteçam deficiências generalizadas de vários nutrientes que têm limitado o crescimento dos Pinus durante toda a rotação.

\section{CONCLUSÃO}

As equações de crescimento testadas para ajustar o crescimento em diâmetro em função da idade apresentaram um bom ajuste e uma alta precisão estatística, permitindo estimar esta variável com boa segurança, tendo se destacado o modelo de Backman modificado. O crescimento em diâmetro das espécies, quando comparados entre si e por nível de degradação do solo, apresentou tendência diferente de níveis e desenvolvimento no tempo, com o Pinus elliottii apresentando melhor desempenho do que o Pinus taeda. O uso de variáveis dummy permitiu definir uma equação única para estimar o crescimento em diâmetro em função da idade para ambas as espécies por níveis de degradação do solo, o que permitiu estratificar o crescimento dessas espécies nestas condições ambientais.

\section{REFERÊNCIAS}

ASSMANN, E. The principle of forest yield study. Oxford: Pergamon, 1970. 506p.

BINKLEY, D. et al. Explaning growth of individual trees: light interception and efficiency of light use by Eucalyptus at four sites in Brazil. Forest Ecology Management, v.259, n.9, p.1704-1713, 2010. Disponível em: <http://www.sciencedirect. com/science/article/pii/S0378112709003910>. Acesso em: 18 mar. 2013. doi:10.1016/j.foreco.2009.05.037.

FREITAS, C.A. et al. O processo de arenização no sudoeste do Rio Grande do Sul: uma alternativa para o seu desenvolvimento sócio-econômico. Disponível em: <http://www.fee.tche.br/eeg/ artigos>. Acesso em: 15 jul. 2003.

GARICOITS, L.S.L. Estado nutricional e fatores do solo limitantes do crescimento de P. taeda L. em Telêmaco Borba
(PR). 1990. 128f. Dissertação (Mestrado em Engenharia Florestal) - Curso de Pós-graduação em Engenharia Florestal, Universidade Federal do Paraná.

GONÇALVES, J.L.M. et al. Relações entre a produtividade de sítios florestais de Eucalyptus grandis e Eucalyptus saligna com as propriedades de alguns solos de textura arenosa e média no Estado de São Paulo. IPEF, n.43/44, p.24-39, 1990.

HANNAH, A. Y. et al. Soil available water as influenced by landscape position and aspect. Agronomy Journal, v.74, p.9991004, 1982.

LOETSCH, F. et al. Forest inventory. Berlim: BLV, 1973. 2v.

LU, D. et al. Linking amazonian secondary succession forest growth to soil properties. Land Degradation \& Development, v.13. n.4, p.331-343, 2002. Disponível em: <http://onlinelibrary. wiley.com/doi/10.1002/ldr.516/pdf>. Acesso em: 27 fev. 2013. doi:10.1002/ldr.516.

MALHI, Y. et al. The above-ground coarse wood productivity of 104 neotropical forest plots. Global Change Biology, v.10, n.5, p.563-591, 2004. Disponível em: <http://onlinelibrary.wiley. com/doi/10.1111/j.1529-8817.2003.00778.x/pdf>. Acesso em: 27 mar. 2013. doi: 10.1111/.1529-8817.2003.00778.x.

MURPHY, P.G.; LUGO, A.E. Ecology of tropical dry forest. Annual Review of Ecology and Systematics, n.17, p.67-88, 1986.

NATH, D.C. et al. Patterns of tree growth in relation to environmental variability in the tropicaldry deciduous forest at Mudumalai, southern India. Journal of Biosciences, v.31, n.5, p.651-669, 2006.

NIMER, E. Clima. In: Geografia do Brasil: Região Sul. Rio de Janeiro: IBGE, 1990. p.151-187.

ONU. Convenção da ONU de combate à desertificação. Nairóbi, Quênia, 17 a 28, outubro, 2005. n.p.

PRODAN, M. et al. Mensura forestal. San José: IICA/ Investigacion y educacion en desarrolo sostenible, 1997. 586p.

REISSMANN, C.B.; WISNEWSKI, C. Aspectos nutricionais de plantios de Pinus. In: GONÇALVES, J.L.M.; BENEDETTI, V. Nutrição e fertilização florestal. Piracicaba: IPEF, 2005. p.135-166.

RICHARDS, F.J. A flexible growth functions for empirical use. Jornal of Experimental Botany, v.10, n.2, p.290-301, 1959.

RUSSO, S.E. et al. Soil-related performance variation and distributions of tree species in a Bornean rain forest. Journal of Ecology, v.93, p.879-889, 2005.

SAS Institute Inc. Sas/Stat user's guide: statistics, Version 9.1.3.ed. Cary, 2004. 1037p. 
SCHNEIDER, P.R. Betriebswirtschaftliche und ertragskundliche grundlagen der forsteinrichtung in sudbrasilien am beispiel von Pinus elliottii. 1984. 190f. Tese (Doutorado em Engenharia Florestal) - Universidade de Freiburg.

SCHNEIDER, P.R. et al. Manual para coleta de informações dendrométricas. Santa Maria: UFSM/CEPER/FATEC, 1988. 28p.

SCHWEINGRUBER, F.H. Tree rings and environment dendroecology. Viena: Haupt, 1996. 609p.

STEEL, R.G.D.; TORRIE, J.H. Principles and procedures of statistics. New York: McGraw, 1960. 481p.
STRECK, E.V. et al. Solos do Rio Grande do Sul. 2.ed. rev. e ampl. Porto Alegre: Emater/RS, 2008. 222p.

SUERTEGARAY, D.M.A. Erosão nos campos sulinos: arenização no sudoeste do Rio Grande do Sul. Revista Brasileira de Geomorfologia, v.12, n.3, p.61-74, 2011.

UNCCD (UNITED NATIONS CONVENTION TO COMBAT DESERTIFICATION INCOUNTRIES). In those Countries Experiencing Serious Drought and/or Desertificacion, Particularly in Africa. Geneve: Interim Secretariat for the Convention to Combat Desertificacion. Geneve Executive Center C.P.76-1219, 1994. 71p. 